ARTICLE

DOI: $10.1038 / \mathrm{s} 41467-018-04321-6$

\title{
Nanographenes as electron-deficient cores of donor-acceptor systems
}

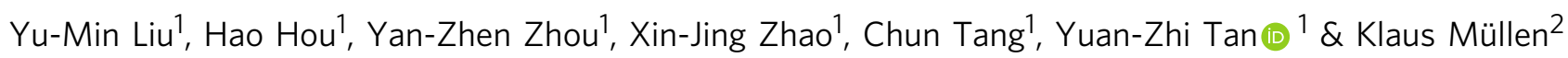

Conjugation of nanographenes (NGs) with electro-active molecules can establish donoracceptor $\pi$-systems in which the former generally serve as the electron-donating moieties due to their electronic-rich nature. In contrast, here we report a series of reversed donoracceptor structures are obtained by $\mathrm{C}-\mathrm{N}$ coupling of electron-deficient perchlorinated NGs with electron-rich anilines. Selective amination at the vertexes of the NGs is unambiguously shown through $\mathrm{X}$-ray crystallography. By varying the donating ability of the anilino groups, the optical and assembly properties of donor-acceptor NGs can be finely modulated. The electron-deficient concave core of the resulting conjugates can host electron-rich guest molecules by intermolecular donor-acceptor interactions and gives rise to charge-transfer supramolecular architectures.

\footnotetext{
${ }^{1}$ Collaborative Innovation Center of Chemistry for Energy Materials, State Key Laboratory for Physical Chemistry of Solid Surfaces, and Department of Chemistry, College of Chemistry and Chemical Engineering, Xiamen University, 361005 Xiamen, China. ${ }^{2}$ Max Planck Institute for Polymer Research, Ackermannweg 10, D-55128 Mainz, Germany. These authors contributed equally: Yu-Min Liu, Hao Hou. Correspondence and requests for materials should be addressed to Y.-Z.T. (email: yuanzhi_tan@xmu.edu.cn)
} 
A s molecularly defined cutouts of the graphene lattice, nanographenes (NGs) have been developed to deduce intrinsic structure-property correlations ${ }^{1-3}$. Among various functional characteristics of NGs, their extended $\mathrm{sp}^{2}$-conjugated carbon skeleton offers intriguing optical absorption, photoluminescence and charge transport properties ${ }^{4-7}$ and thus promising potential in electronics 8,9 and optoelectronics ${ }^{10}$. The electron-rich nature of NGs qualifies them as typical p-type semiconductors in organic field-effect transistors ${ }^{11-14}$ and as electronic donors in organic photovoltaics ${ }^{14-16}$. Allowing conjugation of donor to acceptor moieties has proven as a powerful concept to modulate the optical absorption and charge transport $^{17-22}$. In such donor-acceptor (D-A) conjugates ${ }^{23-25}$, NGs have typically served as donors. Recently, a few studies have switched the electronic characteristics of NGs from electron-rich to electron-poor by multiple introduction of strongly electronwithdrawing groups, such as bisimide ${ }^{26-29}$, chloro $^{30}$, and fluoro $^{31,32}$ substituents. Such electron-deficient NGs thus provide alternative pathway to D-A conjugates. However, this concept remains challenging due to lack of suitable synthetic protocols.

Here we report an amination occurring at the vertexes of perchlorinated NGs by palladium-catalyzed Buchwald-Hartwig coupling ${ }^{33,34}$. Using this strategy, various electron-rich aniline derivatives are coupled to the periphery of electron-deficient chlorinated NGs, providing a series of nano-sized D-A architectures. The concave molecular structures of these D-A NGs are clearly demonstrated by single-crystal X-ray diffraction, which show a selective amination at the vertexes. The donating strength of attached anilino groups can finely tune the optical absorption and intermolecular interactions. Different from previously reported concave polycyclic aromatic hydrocarbons, the concave electron-deficient skeletons of the D-A NGs can assemble with donor-type guest molecules such as tetrathiafulvalene by intermolecular charge transfer (CT).

\section{Results}

Amination of NGs. Arm-chair edges of graphenic structures comprise two types of carbon sites ${ }^{35-37}$, one at the bay and another at the vertex (Fig. 1). The steric hindrance at the bay position is obviously larger than that at the vertex and will become even more severe with bulky groups. In case of perchlorinated NGs, the strong congestion of chloro substituents at the bay has been shown to distort the molecular geometry from planar to curved ${ }^{30}$. We reasoned that the chlorines at the different peripheral sites of NGs possessed different chemical reactivity and could allow selective modification (Fig. 1).

Taking perchlorinated hexa-peri-hexabenzocoronene $\left(\mathrm{C}_{42} \mathrm{Cl}_{18}\right.$, 1, here the smallest representative $\mathrm{NG}$ ) with two types of chloro groups as an initial example, we screened the palladium-catalyzed

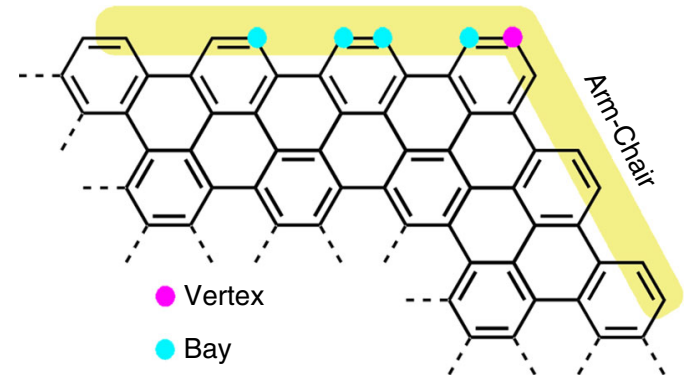

Fig. 1 Arm-chair edge of graphenic structures. Arm-chair edge contains two kinds of carbon sites, one at the bay (cyan point) and another at the vertex (magenta point)
Buchwald-Hartwig C-N cross-coupling between $\mathbf{1}$ and aniline (Fig. 2 and Supplementary Table 1). Under optimized reaction conditions aniline can, indeed, dominantly couple to the vertexes of 1, yielding the hexakis-anilino chlorinated hexa-perihexabenzocoronene (2a) (Fig. 2 and Supplementary Fig. 1). The bulky bidentate phosphine ligand was necessary to produce $\mathbf{2 a}$ (Supplementary Table 1).

In addition to NMR spectroscopy (Supplementary Figs. 9 and 10), the structure of 2 a was assessed by single crystal X-ray diffraction (Fig. 2b). As depicted in Fig. 2b, the most distinguishable feature of $\mathbf{2 a}$ is six substituting anilino groups at the vertexes of NG. The unobstructed remaining chlorines in the bays adopt an up and down conformation, which keeps the structure of 2 a doubly concave (Fig. 2). We further demonstrated that the amination was tolerant to different aniline derivatives, because $\mathbf{2 b}$-2e were synthesized and characterized (Fig. 2 and Supplementary Figs. 4, 7 and 11-18).

Optical properties of 2 . The UV-Vis spectrum of $\mathbf{2 a}$ displays three bands that peak at 452, 482, and $560 \mathrm{~nm}$ (Fig. 2c). The variation of concentration does not affect the absorption profile of $\mathbf{2 a}$, suggesting the absence of the $\pi-\pi$ stacking in solution (Supplementary Fig. 36). The absorption of 2 a showed a bathochromic shift $(45 \mathrm{~nm})$ compared with its parent compound $\mathbf{1}^{30}$, attributed to the intramolecular CT from anilino groups to the inner NG. An expected solvatochromism with increasing solvent polarity was observed for $\mathbf{2 a}$, but the redshift is relatively small (Supplementary Fig. 35).

Comparing the optical absorption, a gradual bathochromic shift of absorption peaks was revealed (Fig. $2 \mathrm{c}$ and Supplementary Table 2) from $\mathbf{2 a}$ to $\mathbf{2 e}$ without concentration dependence (Supplementary Figs. 36-40), in which the electron-donating power of peripheral anilino groups increased. Consequently, the optical HOMO-LUMO gap of $2 \mathbf{a}$ to $2 \mathrm{e}$ decreased from 2.18 to $1.84 \mathrm{eV}$ (Supplementary Table 2). On the other hand, the photoluminescence of compounds $\mathbf{2 a}, \mathbf{2} \mathbf{b}$, and $\mathbf{2 c}$ shows an emission with $\lambda_{\max }$ located at $\sim 560 \mathrm{~nm}$ with an absolute photoluminescence quantum yield (PLQY) of 3.5-5.4\% (Supplementary Table 2), whereas the enhanced intramolecular CT in $\mathbf{2 d}$ and $2 \mathrm{e}$ quenched their photoluminescence dramatically (Supplementary Fig. 41 and Supplementary Table 2). These optical properties of 2 confirmed their D-A characteristics.

Theoretical calculations of 2. Theoretical calculations helped to describe the intramolecular CT in 2. LUMOs were generally distributed over the NG moiety whereas the HOMOs were located at the peripheral anilino units (Supplementary Fig. 52). The electron density differences between the first excitation and the ground state showed a decrease at the anilino units and an increase at the inner NG core (Supplementary Fig. 53) in 2. Differently, the weaker donating ability of anilino groups in 2a leads to poor intramolecular CT, therefore the electron density decrease at the anilino groups of $\mathbf{2 a}$ is obviously smaller than that in 2e (Supplementary Fig. 53), which comprises the electronrichest functional groups. Theoretical and experimental results thus jointly validate the D-A nature of 2 .

Packing structure of 2. The crystal structures of 2a-2e (Supplementary Figs. 31-33 and Supplementary Methods) revealed their assembly in the solid state. All inner cores of $\mathbf{2 a - 2 e}$ have a $C_{3 \mathrm{v}}$ symmetrical double concave structure, if ignoring the conformation of peripheral phenyl groups (Fig. 2b and Supplementary Figs. $31-33$ ), which impedes the $\pi-\pi$ interactions between the NG cores in the solid (Supplementary Fig. 42). Indeed, the solid-state UV-Vis spectra of $\mathbf{2 a - 2 d}$ display small 
a

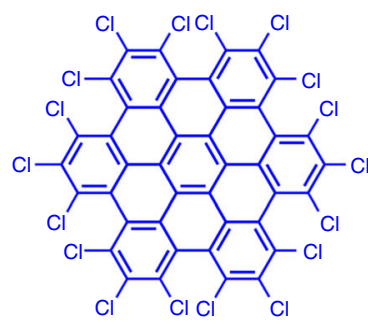

(1)
$\mathrm{Pd}_{2}(\mathrm{dba})_{3}$, BINAP

$\mathrm{RC}_{6} \mathrm{H}_{4} \mathrm{NH}_{2}, \mathrm{Cs}_{2} \mathrm{CO}_{3}$

Toluene, $105^{\circ} \mathrm{C}$

$\mathrm{R}=-\mathrm{H}(\mathbf{2 a}),-\mathrm{iPr}(\mathbf{2 b}),-\mathrm{nBu}(\mathbf{2 c}),-\mathrm{OCH}_{3}(\mathbf{2 d}),-\mathrm{N}\left(\mathrm{CH}_{3}\right)_{2}(\mathbf{2 e})$

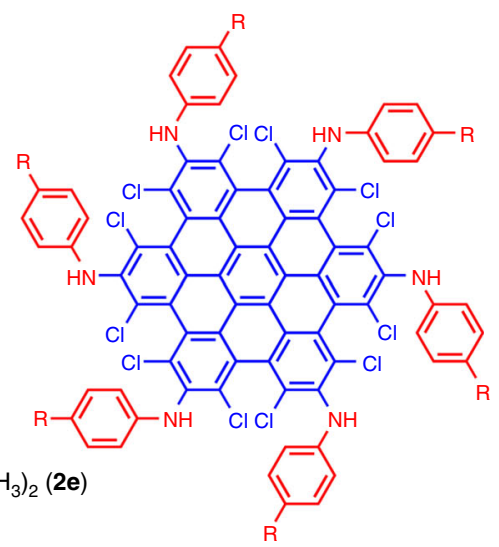

b

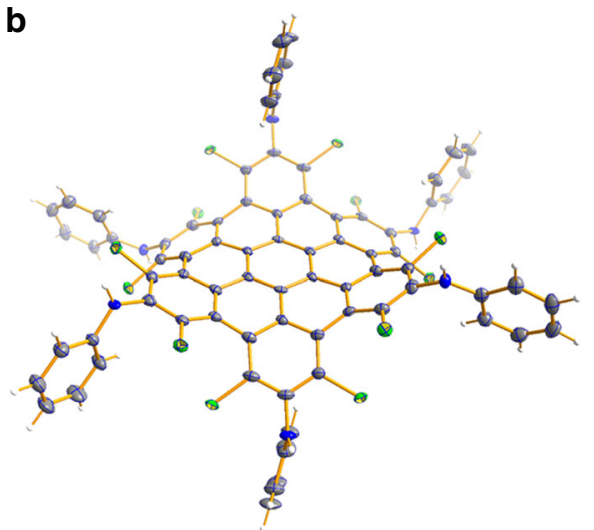

C

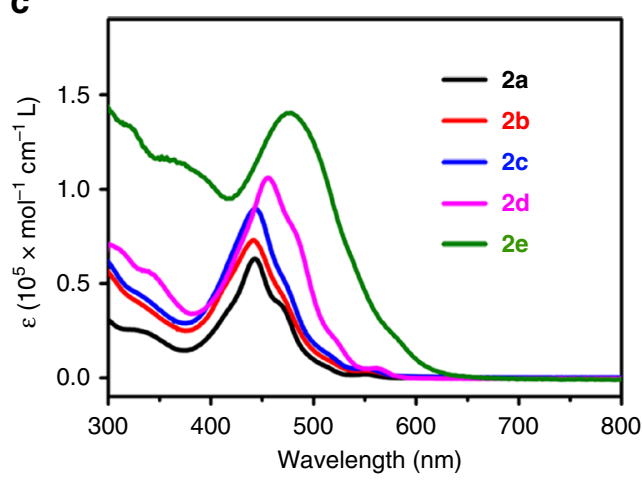

Fig. $\mathbf{2}$ Synthesis and crystal structure of $\mathbf{2}$ and optical properties. a optimized synthetic route for $\mathbf{2}$. $\mathbf{b}$ the doubly concave structure of $\mathbf{2}$ represented by $\mathbf{2 a}$. The thermal ellipsoids are set at $50 \%$ probability level. c UV-Vis absorption spectra of $\mathbf{2}$ measured in dichloromethane (the concentration of $\mathbf{2}$ is $10^{-5}$ $\left.\mathrm{mol} \cdot \mathrm{L}^{-1}\right) \cdot \mathrm{Pd}_{2}(\mathrm{dba})_{3}=$ tris (dibenzylideneacetone)dipalladium. BINAP $=2,2^{\prime}$-bis(diphenylphosphino)-1, $1^{\prime}$-binaphthalene

red shift by 30-35 $\mathrm{nm}$ (Supplementary Figs. 43-46 and Supplementary Methods), in comparison with those in solution, which confirms the absence of $\pi-\pi$ interactions in the solid state for $\mathbf{2 a - 2 d . ~}$

In contrast, in the crystal of $\mathbf{2 e}$, the peripheral p-N-N-dimethyl anilino groups assemble with the skeleton of NG core in a face-toface fashion with a $\pi-\pi$ interaction distance of $3.30 \AA$ (Fig. 3), forming intermolecular D-A supramolecular networks. The different assembly structure of $2 \mathbf{e}$ originates from the stronger donor ability of p-N-N-dimethyl anilino groups, as compared with those in 2a-2d. Remarkably, an obvious red shift by $125 \mathrm{~nm}$ for $2 \mathbf{e}$ appeared in the solid-state optical absorption (Fig. 3), and the absorption of $2 \mathbf{e}$ in the solid extends over the entire range of visible light. The case of $\mathbf{2 e}$ suggests that the intermolecular CT creates an extensive absorption of the D-A molecules, which should be useful for the design of conjugated molecules for light harvesting ${ }^{38}$.

Amination for larger NGs. Encouraged by the successful amination of 1 , we proceeded to larger homologs of $1, \mathrm{C}_{60} \mathrm{Cl}_{22}$ (3) and $\mathrm{C}_{78} \mathrm{Cl}_{26}(5)$, which contain six chlorines at the vertexes as well. Under similar reaction conditions, two series of hexakisaminated chlorinated products $\mathbf{4 a}-\mathbf{4 c}\left(\mathrm{C}_{60} \mathrm{Cl}_{16}\left(\mathrm{NHC}_{6} \mathrm{H}_{4} \mathrm{R}\right)_{6}, \mathrm{R}=\right.$ $-\mathrm{H} \quad(\mathbf{4 a}), \quad-\mathrm{nBu} \quad(\mathbf{4 b})$, and $\left.-\mathrm{OCH}_{3} \quad(\mathbf{4 c})\right)$ and $\mathbf{6 a}-\mathbf{6 b}$ $\left(\mathrm{C}_{78} \mathrm{Cl}_{20}\left(\mathrm{NHC}_{6} \mathrm{H}_{4} \mathrm{R}^{\prime}\right)_{6}, \mathrm{R}^{\prime}=-\mathrm{nBu}(\mathbf{6 a})\right.$ and $\left.-\mathrm{OCH}_{3}(\mathbf{6 b})\right)$ were obtained (Fig. 4 and Supplementary Figs. 2, 5, 6, 8 and 19-30). The unambiguous structure of $\mathbf{4}$ was disclosed by single-crystal $\mathrm{X}$-ray diffraction (Supplementary Methods). The Fig. 4b showed

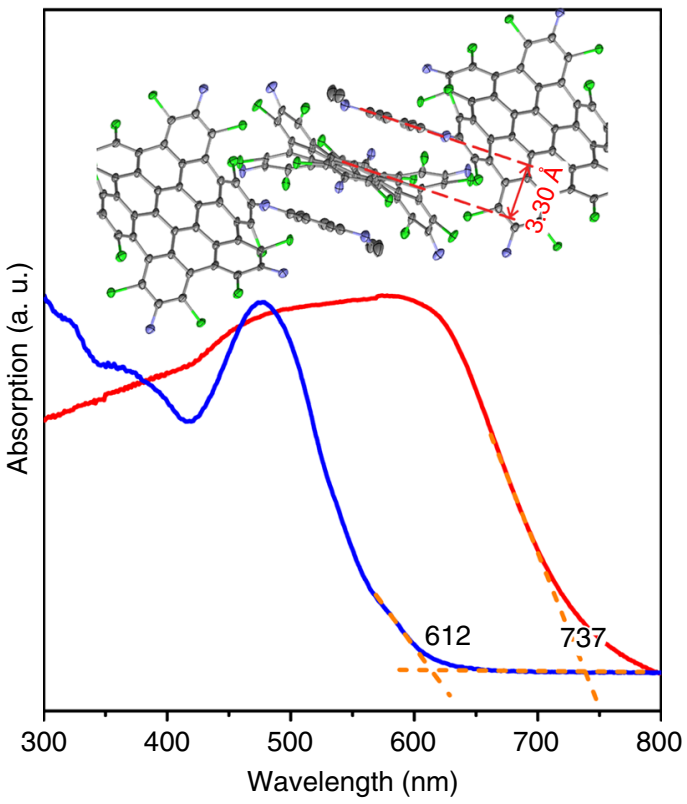

Fig. 3 Absorption and assembly in the solid state of $\mathbf{2 e}$. The UV-Vis absorption of $\mathbf{2 e}$ in solid state (red) measured in a diffuse-reflectance mode was compared with that in dichloromethane (blue, $10^{-5} \mathrm{~mol} \cdot \mathrm{L}^{-1}$ ). The peripheral groups which do not allow D-A interactions with the inner NG were omitted for clarity 
a

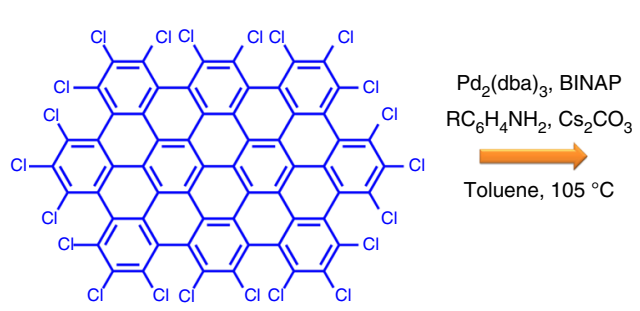

(3)
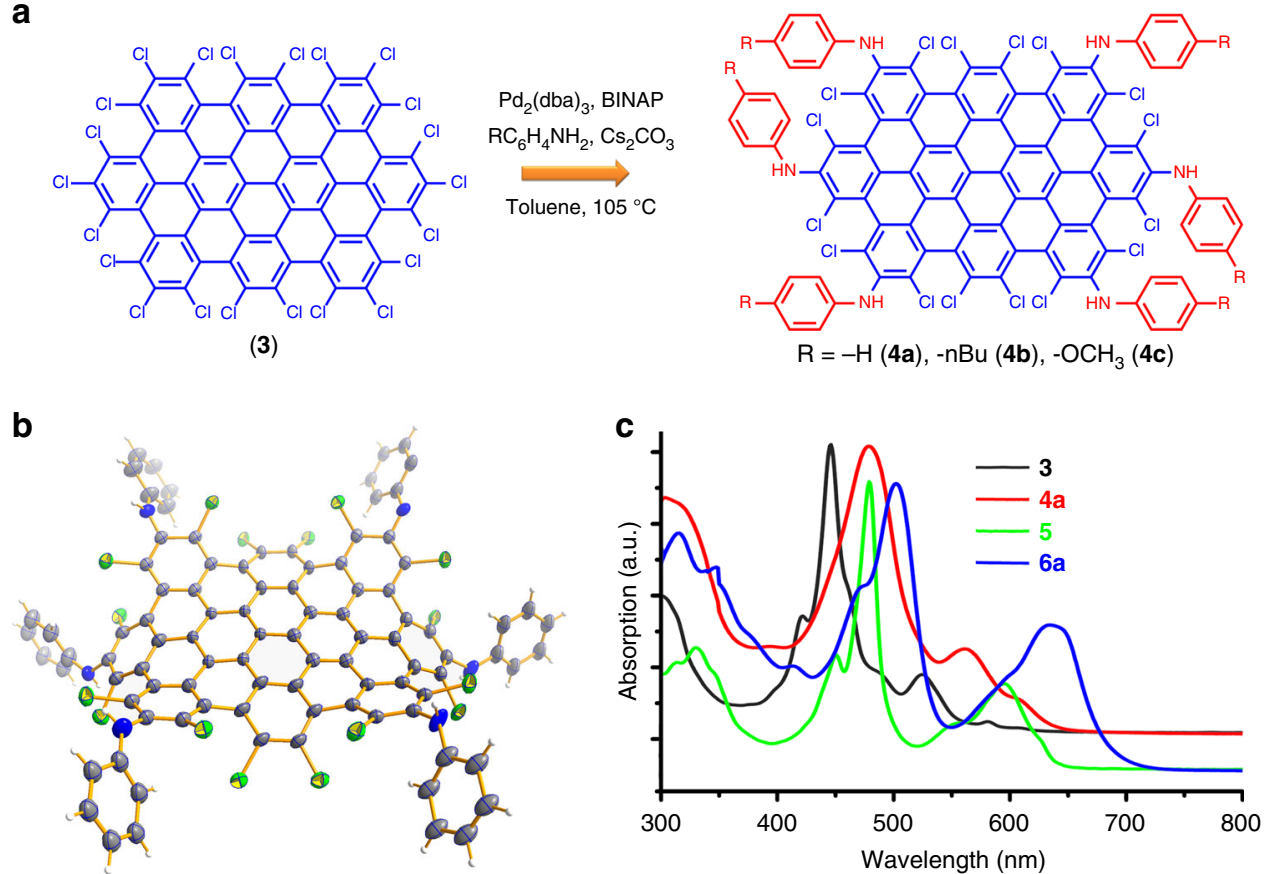

Fig. 4 Synthesis and crystal of $\mathbf{4}$ and optical absorption. a optimized synthetic route for $\mathbf{4}$. $\mathbf{b}$ the crystal structure of $\mathbf{4}$ represented by $\mathbf{4 a}$. The thermal ellipsoids were set at $30 \%$ probability level. c UV-Vis absorption spectra of $\mathbf{3}, \mathbf{4 a}, \mathbf{5}$, and $\mathbf{6} \mathbf{a}$. Notably, the molar absorption coefficients of $\mathbf{3}, \mathbf{4}$, and $\mathbf{6}$ were listed in Supplementary Figs. 47-49, while that of $\mathbf{5}$ can not be measured due to its extremely low solubility

that six anilino groups are indeed connected to the vertexes of 3 while the chlorines at the bay again keep intact. The UV-Vis spectra of $\mathbf{4}$ and $\mathbf{6}$ display bathochromic shifts by 39 and $37 \mathrm{~nm}$ (Fig. 4c, Supplementary Figs. 47-49 and Supplementary Table 3), respectively, compared with 3 and 5, also due to the intramolecular CT. By theoretical calculations, 4 exhibits a typical distribution of electron density differences as a feature of D-A conjugates (Supplementary Fig. 55).

Supramolecular assembly between D-A NGs and tetrathiafulvalene. D-A NGs own an electron-deficient concave core, which can act as supramolecular host for electron-rich donor molecules such as tetrathiafulvalene (TTF). Evaporation of the carbon disulfide solution of $\mathbf{2 a}$ and TTF led to the formation of crystalline black needles, different from the orange prisms of $\mathbf{2 a}$. $\mathrm{X}$-ray diffraction analysis revealed that a supramolecular complex 2a $\supset$ TTF had formed with TTF molecules located at the center of concave NG in a face-to-face manner (Fig. 5a, c). The interfacial distance between TTF and NG core is $3.30 \AA$ implying strong $\pi-\pi$ interactions. Further stacking between TTF and $\mathbf{2 a}$ builds up a mixed D-A-D-A supramolecular column (Fig. $5 \mathrm{c}$ ). The columnar supramolecular architecture of $2 \mathrm{a} \supset$ TTF holds promise for long-range orientations of CT dipoles, making it a potential organic ferroelectric ${ }^{39,40}$. Compound 4 as acceptor can also host electron-donating TTF (Fig. 5 and Supplementary Fig. 34). One TTF molecule is located above the core of 4 , forming sandwich-type complexes (2.4 つTTF) with close interfacial $\pi-\pi$ interactions $(3.30 \AA$ ) between TTF and inner NG.

The structures of both supramolecular complexes (2a $\supset$ TTF and 2.4 つTTF) suggest the presence of intermolecular CT between TTF and electronic deficient core as proven by CT bands in the absorption spectra of $2 \mathrm{a} \supset$ TTF and $2.4 \mathrm{JTF}$ (Fig. 5). In comparison with $\mathbf{2 a} \supset \mathrm{TTF}$, a bathochromic shift $(130 \mathrm{~nm})$ appeared in the CT band of 2.4 כ TTF (Fig. 5f and
Supplementary Fig. 50), attributed to the extended conjugated skeleton of 4. Remarkably, the absorption of 2.4 つ TTF crystals can extend into the NIR region up to $1000 \mathrm{~nm}$. The unambiguous structure and clear CT band of the supramolecular complexes between D-A NGs and donating guests (Fig. 5) characterized these D-A NG conjugates as promising electronicaccepting host for the construction of CT supramolecular architectures.

\section{Discussion}

Our amination protocol enabled functionalization at the vertexes of NGs and constructed reversed D-A conjugates. The donating ability of aniline groups affords an efficient approach to modulate the properties of D-A NGs, e.g., absorption, optical gap, luminescence, and assembly. Since the edge halogenation of graphene nanoribbons and graphenic materials was achieved, our functionalization concept can additionally incorporate D-A structures and CT dipoles into these graphenic structures. The resulting D-A NGs undergo assembly with electron-rich molecules by intermolecular D-A interactions, creating CT supramolecular architectures. This opens an avenue toward supramolecular functional materials based on NG acceptors.

\section{Methods}

Synthesis of 2. First, the reaction conditions, including amount of aniline, phosphine ligands and bases, for the palladium-catalyzed Buchwald-Hartwig C-N coupling between 1 and aniline were screened (Supplementary Table 1). The optimized conditions required a large excess of aniline to favor the multiple C-N coupling of 1 with aniline and suppress the homocoupling between $1^{41,42}$, supported by the control experiments (Supplementary Table 1). The decreased amount of aniline in control experiments resulted in a lower yield of $2 \mathbf{a}$, whereas the experiments with 15 and 30 fold excess of aniline did not affect the yield (Supplementary Table 1).

Under the optimized reaction conditions, a $25 \mathrm{ml}$ reaction tube was charged with 1 (100 mg, $0.087 \mathrm{mmol}$ ), $\mathrm{Cs}_{2} \mathrm{CO}_{3}$ (113 mg, $0.348 \mathrm{mmol}$ ), $\mathrm{Pd}_{2}(\mathrm{dba})_{3},(24 \mathrm{mg}, 0.026$ $\mathrm{mmol}$ ) and BINAP (32 mg, $0.052 \mathrm{mmol})$ under argon, and then an aniline (194 mg, 
a

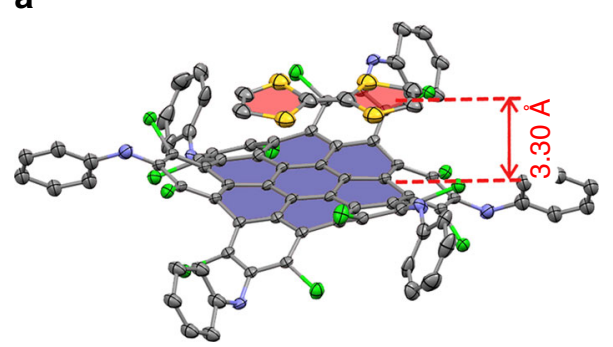

C

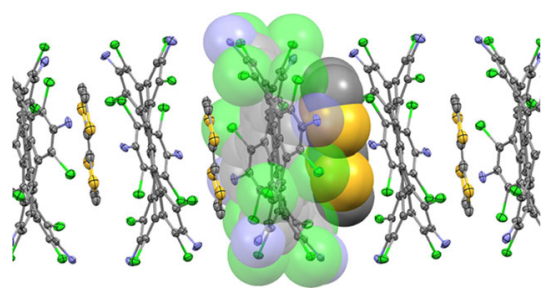

e

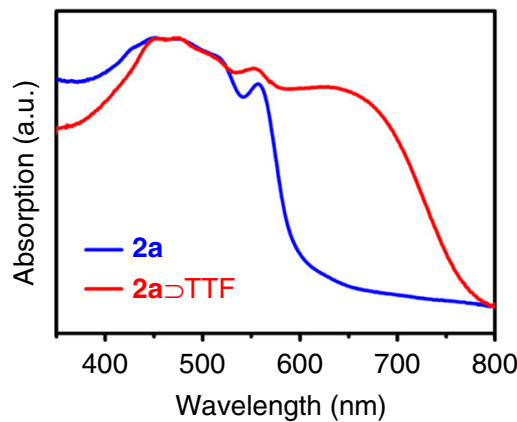

b

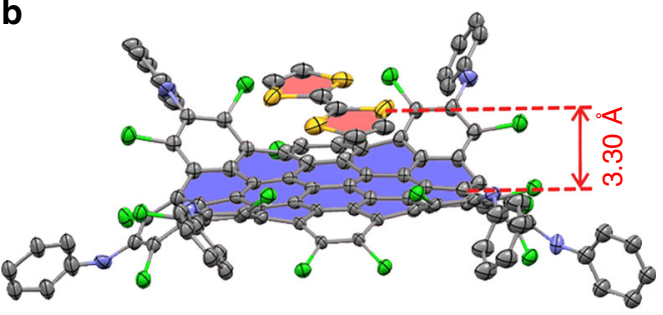

d

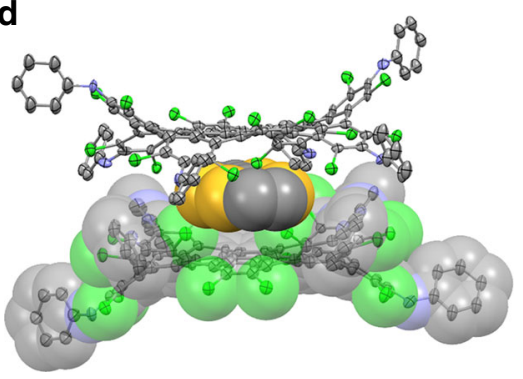

$\mathbf{f}$

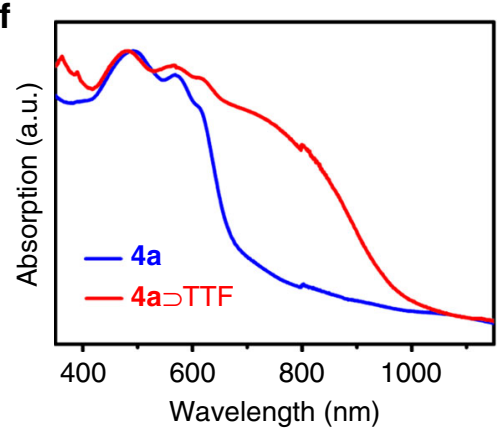

Fig. 5 Supramolecular assembly between D-A NGs and TTF. $\mathbf{a}, \mathbf{b}$ the structures of $\mathbf{2 a} \supset$ TTF and $\mathbf{4 a} \supset$ つTF. $\mathbf{c}$ one dimensional mixed-stacking of $\mathbf{2 a}$ and TTF in the crystal. Peripheral phenyl groups were omitted for clarity. $\mathbf{d}$ sandwich-type complex of 2.4a $\supset$ TTF. e, $\mathbf{f} U V$-Vis-NIR spectra of $\mathbf{2 a}, \mathbf{2 a} \supset$ TTF, $\mathbf{4 a}$ and 2.4a $\supset$ TTF in the solid state measured in a diffuse-reflectance mode. CT bands at $650 \mathrm{~nm}$ and $780 \mathrm{~nm}$ were clearly observed in the spectra of supramolecular complex

$2.09 \mathrm{mmol}$, equal to 4 -fold according to the molar of chlorine at the vertexes) solution in toluene $(5 \mathrm{ml})$ was added into the tube. The reactants were stirred and refluxed at $105^{\circ} \mathrm{C}$ for $36 \mathrm{~h}$. After the reaction, the products were extracted with dichloromethane (DCM) $(30 \mathrm{ml})$ and washed with water $(20 \mathrm{ml})$. The collected organic phase was dried by anhydrous $\mathrm{MgSO}_{4}$. After filtration and evaporation of the solvent, the crude products were separated over a silica column using DCM/ petroleum ether $\left(60-90^{\circ} \mathrm{C}\right)(1.5: 1)$ as eluent. The red component was collected, yielding $40 \mathrm{mg}$ of $\mathbf{2 a}$. The yield was $31 \%$ (Note that the yield of $\mathbf{2 a}$ determined by NMR spectroscopy is $\sim 50 \%$, Supplementary Table 1 ). Due to strong absorption of $\mathbf{2 a}$ on silica, the subsequent eluent contained $\mathbf{2 a}$ as well (Supplementary Fig. 1). If these components (spot 3 shown in Supplementary Fig. 1) were collected and separated again, the isolated yield can be improved to $38 \%$.

Besides 2a as the major product of $\mathrm{C}-\mathrm{N}$ coupling, the byproducts in the reaction were attributed to the oligomers originating from the competitive palladium-catalyzed $\mathrm{C}-\mathrm{C}$ homocoupling between $\mathbf{1}^{41,42}$ and a few pentakis-anilino products. The byproducts with more than 6 anilino groups were not observed. Considering that six $\mathrm{C}-\mathrm{N}$ bonds are formed in one reaction, the achieved yield is over $80 \%$ for each $\mathrm{C}-\mathrm{N}$ bond formation.

$\mathbf{2 b}, \mathbf{2 c}, \mathbf{2 d}$, and $\mathbf{2 e}$ were synthesized by the coupling of $\mathbf{1}$ to different aniline derivatives employing similar reaction and separation conditions. The isolated yields of $\mathbf{2 b}, \mathbf{2 c}, \mathbf{2 d}$, and $\mathbf{2 e}$ were $32,26,26$, and $21 \%$, respectively.

Synthesis of 4. A $25 \mathrm{ml}$ reaction tube was charged with $3(50 \mathrm{mg}, 0.033 \mathrm{mmol})$ $\mathrm{Cs}_{2} \mathrm{CO}_{3}$ (43 mg, $\left.0.133 \mathrm{mmol}\right), \mathrm{Pd}_{2}(\mathrm{dba})_{3}(9 \mathrm{mg}, 0.01 \mathrm{mmol})$ and BINAP (12 mg, $0.02 \mathrm{mmol}$ ) under argon. An aniline $(74 \mathrm{mg}, 0.792 \mathrm{mmol}$, equal to 4 -fold according to the molar of chlorine at the vertexes of 3$)$ solution in toluene $(5 \mathrm{ml})$ was added into the tube. After stirring at $105^{\circ} \mathrm{C}$ for $36 \mathrm{~h}, 30 \mathrm{ml} \mathrm{DCM}$ was added. The organic phase was washed with water $(20 \mathrm{ml})$ and dried over anhydrous $\mathrm{MgSO}_{4}$. After removing the solvent, the crude products were separated by a silica column using $\mathrm{DCM} /$ petroleum ether $\left(60-90^{\circ} \mathrm{C}\right)(1.5: 1)$ as eluent. The collected component was further purified by high performance liquid chromatography (HPLC) using JAIGEL-2H column (Japan Analytical Industry Ltd., chloroform as eluent), yielding $10 \mathrm{mg}$ of $\mathbf{4 a}$. The yield was $17 \%$.

$\mathbf{4 b}$, and $\mathbf{4 c}$ were also synthesized using different aniline derivatives under similar reaction and separation conditions as those of $\mathbf{4 a}$. Notably, purification by HPLC using JAIGEL-2H column was necessary for $\mathbf{4 b}$ and $\mathbf{4 c}$ as well. The isolated yields of $\mathbf{4 b}$ and $\mathbf{4 e}$ were 13 and $20 \%$, respectively, under the optimized conditions.

Synthesis of 6.5 ( $50 \mathrm{mg}, 0.027 \mathrm{mmol}), \mathrm{Cs}_{2} \mathrm{CO}_{3}(36 \mathrm{mg}, 0.133 \mathrm{mmol}), \mathrm{Pd}_{2}(\mathrm{dba})_{3}$ ( $8 \mathrm{mg}, 0.0099 \mathrm{mmol})$, and BINAP (10 $\mathrm{mg}, 0.0198 \mathrm{mmol}$ ) were introduced into a 25 $\mathrm{ml}$ reaction tube under argon (Supplementary Fig. 2). Then toluene $(5.0 \mathrm{ml})$ and 4 n-butyl-aniline $(96 \mathrm{mg}, 0.792 \mathrm{mmol}$, equal to a 4 -fold according to the molar of chlorine at the vertexes) were added. The reactants were stirred for $36 \mathrm{~h}$ at $105^{\circ} \mathrm{C}$. The products were extracted with DCM $(30 \mathrm{ml})$, washed with water $(20 \mathrm{ml})$ and dried over anhydrous $\mathrm{MgSO}_{4}$. After removing the solvent, the crude products were separated over a silica column using DCM/petroleum ether $\left(60-90^{\circ} \mathrm{C}\right)(1: 3)$ as the eluent. Then, the obtained component was further purified by HPLC using JAIGEL-2H column (chloroform as the eluent), yielding $13 \mathrm{mg}$ of $\mathbf{6 a}$. The yield was $19 \%$. Similarly, $\mathbf{6 b}$ was synthesized and isolated, with a yield of $8 \%$.

Crystallography. Using Olex $2^{43}$, all the initial structures were solved with the SHELX-XT structure solution program by the direct method and refined with the XL refinement package by Least Squares minimization. The crystals of $4 a$ and $4 c$ easily effloresced, thus the structures of $4 \mathbf{a}$ and $4 \mathbf{c}$ were obtained from the crystal structures of their respective supramolecular complexes. For compound $\mathbf{6}$, single crystals could be obtained which were too tiny to be measured by X-ray diffraction.

Theoretical calculations. All the calculations were performed with the Gaussian 09 software package ${ }^{44}$. The solvent effects of DCM used in experiment have been 
considered using the CPCM continuum solvation model ${ }^{45}$. First, in order to ensure the suitable hybrid functional, we compared the theoretical HOMO-LUMO gap calculated by B3LYP, CAM-B3LYP, M062X, and HSEH1PBE ${ }^{46}$ with the experimental optical HOMO-LUMO gap (Supplementary Table 4). The optimized structures by different functionals were also compared with the experimental crystallographic data, taking 2a as an example (Supplementary Fig. 51, Supplementary Table 5). Accordingly, the HSEH1PBE functional is more appropriate for the theoretical calculations (Supplementary Figs. 52-55). Then we calculated the electron density differences between the first excitation state (corresponding to the UV-Vis absorption with maximum wavelength and mainly arising from HOMO to LUMO transition) and the ground state for compounds $\mathbf{2 - 4}$ by HSEH1PBE.

Data availability. Supplementary crystallographic data for this manuscript have been deposited at the Cambridge Crystallographic Data Centre under deposition numbers CCDC 1580856-1580862. These data can be obtained free of charge from [http://www.ccdc.cam.ac.uk/data_request/cif]. The authors declare that all the data supporting the findings of this study are available within the article (and Supplementary Information File), or available from the corresponding author on reasonable request.

Received: 12 December 2017 Accepted: 19 April 2018

Published online: 15 May 2018

\section{References}

1. Narita, A., Wang, X.-Y., Feng, X. \& Müllen, K. New advances in nanographene chemistry. Chem. Soc. Rev. 44, 6616-6643 (2015).

2. Müllen, K. Evolution of graphene molecules: structural and functional complexity as driving forces behind nanoscience. ACS Nano 8, 6531-6541 (2014).

3. Segawa, Y., Ito, H. \& Itami, K. Structurally uniform and atomically precise carbon nanostructures. Nat. Rev. Mater. 1, 15002 (2016).

4. Stepien, M., Gonka, E., Zyla, M. \& Sprutta, N. Heterocyclic nanographenes and other polycyclic heteroaromatic compounds: synthetic routes, properties, and applications. Chem. Rev. 117, 3479-3716 (2017).

5. Wu, J., Pisula, W. \& Müllen, K. Graphenes as potential material for electronics. Chem. Rev. 107, 718-747 (2007).

6. Vo, T. H. et al. Large-scale solution synthesis of narrow graphene nanoribbons. Nat. Commun. 5, 3189 (2014).

7. Mehdi Pour, M. et al. Laterally extended atomically precise graphene nanoribbons with improved electrical conductivity for efficient gas sensing. Nat. Commun. 8, 820-820 (2017).

8. Cao, J. et al. Well-defined thiolated nanographene as hole-transporting material for efficient and stable perovskite solar cells. J. Am. Chem. Soc. 137, 10914-10917 (2015)

9. Abbas, A. N. et al. Vapor-phase transport deposition, characterization, and applications of large nanographenes. J. Am. Chem. Soc. 137, 4453-4459 (2015).

10. Paterno, G. M. et al. Synthesis of dibenzo hi, st ovalene and its amplified spontaneous emission in a polystyrene matrix. Angew. Chem. Int. Ed. 56, 6753-6757 (2017).

11. Woehrle, T. et al. Discotic liquid crystals. Chem. Rev. 116, 1139-1241 (2016).

12. Sakaguchi, H., Song, S., Kojima, T. \& Nakae, T. Homochiral polymerizationdriven selective growth of graphene nanoribbons. Nat. Chem. 9, 57-63 (2017).

13. Matsuo, K., Saito, S. \& Yamaguchi, S. A soluble dynamic complex strategy for the solution-processed fabrication of organic thin-film transistors of a boroncontaining polycyclic aromatic hydrocarbon. Angew. Chem., Int. Ed. 55, 11984-11988 (2016).

14. Xiao, S. et al. Controlled doping in thin-film transistors of large contorted aromatic compounds. Angew. Chem., Int. Ed. 52, 4558-4562 (2013).

15. Loh, K. P., Tong, S. W. \& Wu, J. Graphene and graphene-like molecules: prospects in solar cells. J. Am. Chem. Soc. 138, 1095-1102 (2016).

16. Kang, S. J. et al. A supramolecular complex in small-molecule solar cells based on contorted aromatic molecules. Angew. Chem. Int. Ed. 51, 8594-8597 (2012).

17. Teran, N. B. et al. Twisted thiophene-based chromophores with enhanced intramolecular charge transfer for cooperative amplification of third-order optical nonlinearity. J. Am. Chem. Soc. 138, 6975-6984 (2016).

18. Kast, H. et al. Acceptor-substituted S,N-heteropentacenes of different conjugation length: structure-property relationships and solar cell performance. Adv. Funct. Mater. 25, 3414-3424 (2015).

19. Haid, S. et al. Significant improvement of dye-sensitized solar cell performance by small structural modification in pi-conjugated donor-acceptor dyes. $A d v$. Funct. Mater. 22, 1291-1302 (2012).
20. Mishra, A., Fischer, M. K. R. \& Baeuerle, P. Metal-free organic dyes for dyesensitized solar cells: from structure: property relationships to design rules. Angew. Chem. Int. Ed. 48, 2474-2499 (2009).

21. Zhang, X. et al. Dithienopyrrole-based donor-acceptor copolymers: low bandgap materials for charge transport, photovoltaics and electrochromism. J. Mater. Chem. 20, 123-134 (2010).

22. Steckler, T. T. et al. A spray-processable, low bandgap, and ambipolar donoracceptor conjugated polymer. J. Am. Chem. Soc. 131, 2824-2826 (2009).

23. Mativetsky, J. M. et al. Self-assembly of a donor-acceptor dyad across multiple length scales: functional architectures for organic electronics. Adv. Funct. Mater. 19, 2486-2494 (2009).

24. Wong, W. W. H. et al. Self-assembling thiophene dendrimers with a hexaperi-hexabenzocoronene core-synthesis, characterization and performance in bulk heterojunction solar cells. Chem. Mater. 22, 457-466 (2010).

25. Keerthi, A. et al. Hexa-peri-hexabenzocoronene with different acceptor units for tuning optoelectronic properties. Chem. Asian J. 11, 2710-2714 (2016)

26. Zyla-Karwowska, M. et al. An electron-deficient azacoronene obtained by radial pi extension. Angew. Chem. Int. Ed. 55, 14658-14662 (2016).

27. Seifert, S., Shoyama, K., Schmidt, D. \& Wuerthner, F. An electron-poor C64 nanographene by palladium-catalyzed cascade C-C bond formation: one-pot synthesis and single-crystal structure analysis. Angew. Chem., Int. Ed. 55, 6390-6395 (2016).

28. Zhong, Y. et al. Helical ribbons for molecular electronics. J. Am. Chem. Soc. 136, 8122-8130 (2014).

29. Zhong, Y. et al. Molecular helices as electron acceptors in high-performance bulk heterojunction solar cells. Nat. Commun. 6, 8242 (2015).

30. Tan, Y.-Z. et al. Atomically precise edge chlorination of nanographenes and its application in graphene nanoribbons. Nat. Commun. 4, 3646 (2013).

31. Loo, Y.-L. et al. Unusual molecular conformations in fluorinated, contorted hexabenzocoronenes. Org. Lett. 12, 4840-4843 (2010).

32. Mori, T., Kikuzawa, Y. \& Takeuchi, H. N-type field-effect transistor based on a fluorinated-graphene. Org. Electron. 9, 328-332 (2008).

33. Ruiz-Castillo, P. \& Buchwald, S. L. Applications of palladium-catalyzed C-N cross-coupling reactions. Chem. Rev. 116, 12564-12649 (2016).

34. Park, Y., Kim, Y. \& Chang, S. Transition metal-catalyzed C-H amination: Scope, mechanism, and applications. Chem. Rev. 117, 9247-9301 (2017).

35. Bellunato, A., Tash, H. A., Cesa, Y. \& Schneider, G. F. Chemistry at the edge of graphene. Chemphyschem 17, 785-801 (2016).

36. Eigler, S. \& Hirsch, A. Chemistry with graphene and graphene oxidechallenges for synthetic chemists. Angew. Chem. Int. Ed. 53, 7720-7738 (2014).

37. Hirsch, A., Englert, J. M. \& Hauke, F. Wet chemical functionalization of graphene. Acc. Chem. Res. 46, 87-96 (2013).

38. Vezie, M. S. et al. Exploring the origin of high optical absorption in conjugated polymers. Nat. Mater. 15, 746-753 (2016).

39. Tayi, A. S. et al. Room-temperature ferroelectricity in supramolecular networks of charge-transfer complexes. Nature 488, 485-489 (2012).

40. Tayi, A. S., Kaeser, A., Matsumoto, M., Aida, T. \& Stupp, S. I. Supramolecular ferroelectrics. Nat. Chem. 7, 281-294 (2015).

41. Hassan, J., Sevignon, M., Gozzi, C., Schulz, E. \& Lemaire, M. Aryl-aryl bond formation one century after the discovery of the Ullmann reaction. Chem. Rev. 102, 1359-1470 (2002).

42. Wang, L., Zhang, Y., Liu, L. \& Wang, Y. Palladium-catalyzed homocoupling and cross-coupling reactions of aryl halides in poly(ethylene glycol). J. Org. Chem. 71, 1284-1287 (2006).

43. Dolomanov, O. V., Bourhis, L. J., Gildea, R. J., Howard, J. A. K. \& Puschmann, H. OLEX2: a complete structure solution, refinement and analysis program. $J$. Appl. Cryst. 42, 339-341 (2009).

44. Frisch, M. J. et al. Gaussian 09, Revision A.02 Gaussian Inc. (2016).

45. Barone, V. \& Cossi, M. Quantum calculation of molecular energies and energy gradients in solution by a conductor solvent model. J. Phys. Chem. A 102, 1995-2001 (1998).

46. Jacquemin, D., Planchat, A., Adamo, C. \& Mennucci, B. TD-DFT assessment of functionals for optical 0-0 transitions in solvated dyes. J. Chem. Theory Comput. 8, 2359-2372 (2012).

\section{Acknowledgements}

This work was financially supported by the National Natural Science Foundation of China $(21771155,21721001)$, the Ministry of Science and Technology of China (2014CB845603, 2017YFA0204902), the Thousand Youth Talents Plan and the Fundamental Research Funds for the Central Universities (20720180035). We appreciated Prof. Zexing Cao and Mr. Mingjun Sun for the help on the theoretical calculations.

\section{Author contributions}

K.M. and Y-Z.T. conceived and designed the experiments; Y.-M.L, H.H, Y.-Z.Z., and X.J.Z. conducted synthesis and completed the identification; C.T. performed the theoretical 
work; Y.-M.L., Y.-Z.T., and K.M. co-wrote the paper; All authors discussed the results and commented on the manuscript.

\section{Additional information}

Supplementary Information accompanies this paper at https://doi.org/10.1038/s41467018-04321-6.

Competing interests: The authors declare no competing interests.

Reprints and permission information is available online at http://npg.nature.com/ reprintsandpermissions/

Publisher's note: Springer Nature remains neutral with regard to jurisdictional claims in published maps and institutional affiliations. (c) (i) Open Access This article is licensed under a Creative Commons Attribution 4.0 International License, which permits use, sharing, adaptation, distribution and reproduction in any medium or format, as long as you give appropriate credit to the original author(s) and the source, provide a link to the Creative Commons license, and indicate if changes were made. The images or other third party material in this article are included in the article's Creative Commons license, unless indicated otherwise in a credit line to the material. If material is not included in the article's Creative Commons license and your intended use is not permitted by statutory regulation or exceeds the permitted use, you will need to obtain permission directly from the copyright holder. To view a copy of this license, visit http://creativecommons.org/ licenses/by/4.0/.

(C) The Author(s) 2018 